\title{
Implementación de la plataforma GOOGLE CLASROOM en la asignatura "Tratamiento de Residuos" para la realización de experiencia de clase inversa.
}

\section{Samper ${ }^{a}$, D. Garcia-Garcia ${ }^{a}$, JM Ferri ${ }^{a}$, A. Carbonell-Verdu ${ }^{a}$ y J. Lopez-Martinez ${ }^{a}$}

${ }^{a}$ UniversitatPolitècnica de València. Departamento de Ingeniería Mecánica y de Materiales. PlazaFerrándiz y Carbonell, 03801 Alcoy (Alicante) España. e-mail: masammad@epsa.upv.es

\begin{abstract}
During the academic year 2016-17 the flipped classroom methodology was implemented using the GOOGLE CLASS platform in the "waste treatment" subject. This methodology has allowed students to have more time in the classroom to solve doubts and practical problems, increasing collaborative work. Using this methodology, the self-assessment test score increased compared to other years and students prefer this methodology to the traditional master class as indicated in an anonymous survey.
\end{abstract}

Keywords: methodology, flipped classroom, self-assessment.

\begin{abstract}
Resumen
Durante el curso académico 2016-17 se ha implantado la metodología de clase inversa utilizando la plataforma GOOGLE CLASSROOM en la asignatura "Tratamiento de Residuos". Esta metodología ha permitido que los alumnos dispongan de más tiempo en el aula para resolver dudas y realizar prácticas, aumentando el trabajo colaborativo. Mediante esta metodología la nota media del test de autoevaluación subió respecto a otros años y los alumnos prefieren está metodología a la tradicional clase magistral según indicaron en una encuesta anónima.
\end{abstract}

Palabras clave: metodología, clase invertida, autoevaluación. 


\section{Introducción}

Todas las personas son conscientes del cambio exponencial que representa el uso de la tecnología en la vida cotidiana. Especialmente, los jóvenes de hoy pasan la mayoría de las horas del día en contacto con diferentes aparatos electrónicos, desde móviles de última generación (smartphones), pasando por dispositivos tipo tablets hasta los más convencionales, pero no menos importantes, ordenadores. Esto se debe principalmente al modo de obtener la información, que ha ido evolucionando, pasando de la obtención de la información a través de libros y por medio de las clases magistrales que impartían los docentes a la obtención de información instantánea que nos ofrece internet.

Si bien es verdad que esto es un indicador del desarrollo de la población en diferentes ámbitos (tecnológico, bienestar, etc), no deja de ser un problema en otros ámbitos como la enseñanza. El modelo estandarizado de la enseñanza universitaria se basa en una gran carga lectiva donde más de un $60 \%$ de las horas que se imparten en una asignatura son de clases donde se explican conceptos teóricos y el resto se reparte entre prácticas de laboratorio (para el caso de grados en ingeniería o grados de ciencias) y trabajos a realizar en casa. Por tanto, el alumnado de hoy, presenta niveles de concentración muy bajos, en asignaturas cuyo contenido teórico es elevado, ya que se están acostumbrando a aprender mediante el uso de esta tecnología. Es por ello que la investigación sobre la metodología de impartición docente debe crear nuevos modelos de enseñanza donde se priorice el uso de este tipo de tecnologías, sabiendo que este es el medio por el que el alumnado presta mayor atención.

Desde hace muy pocos años autores como Jonathan Bergmann y Aaron Sams (Bergmann,2012) basaron sus clases haciendo que el alumnado cooperara más durante el tiempo de clase y dejaban ciertos aprendizajes de tipo conceptual, como tareas para realizar en casa. Concretamente, el docente realizaba en el horario lectivo tareas de tipo práctico a sabiendas que los alumnos ya habían realizado las tareas de casa (tipo conceptual) y se aprovechaba para reforzar dichos conceptos de una forma aplicada. A esta metodología innovadora le llamaron "clase inversa" (Barrera, 2013) (Jordan-Lluch, 2014). El material teórico-conceptual era subido a una plataforma virtual por el docente en formato digital, donde se podía encontrar material tipo videos explicativos, chats interactivos, documentos, etc.

En el presente trabajo, se ha aplicado la metodología "clase inversa" a la asignatura "Tratamiento de Residuos" impartida en el Grado de Ingeniería Química y se ha usado "GOOGLE CLASSROOM" como plataforma virtual donde poder subir material teórico-conceptual en formato video (Gálvez, 2015) (López Rodríguez,2016), mensajería para poder resolver dudas particulares con el alumno, exámenes para realizar autoevaluaciones incluso para realizar el examen final de la asignatura (Tourón, 2014). El propósito final de este estudio fue el de valorar las posibles mejoras de la metodología aplicada y el grado de atención del alumnado como clave para mejorar los resultados académicos, evaluar de forma continua la implicación. Finalmente se hizo una encuesta al alumnado para evaluar el índice de aceptación del método con respecto a los métodos tradicionales a los que estaban habituados.

\section{Objetivos}

El principal objetivo del presente trabajo es el empleo y evaluación de la plataforma GOOGLE CLASSROOM para la interacción alumno-profesor en las clases de "Tratamiento de Residuos" de cuarto curso del Grado de Ingeniería Química. Se ha decidido la implementación de dicha plataforma con el fin de agilizar y mejorar dicha interacción entre los integrantes de la clase facilitando de esta forma el desarrollo y la aplicación de la metodología de clase inversa para el desarrollo de las mismas. Ya que mediante dicha metodología es el alumno el que debe adquirir los conocimientos de forma autónoma con ayuda del material aportado por el profesor previamente a las clases presenciales dejando las sesiones lectivas para fomentar el aprendizaje práctico y la cooperación entre los alumnos, es por ello que la 
plataforma GOOGLE CLASSROOM es una buena alternativa para dotar a los alumnos del material necesario para su autoaprendizaje.

\section{Desarrollo}

A continuación se desarrollará la experiencia con el empleo de GOOGLE CLASSROOM en el desarrollo de la asignatura de "Tratamiento de Residuos" impartida mediante metodología inversa. Para la implantación de dicha metodología es necesario un trabajo previo por parte del profesor así como la colaboración activa por parte del alumnado, ya que dicha aplicación permite una continua comunicación entre alumno-profesor, y alumno-alumno, permitiendo la generación de debates sobre la asignatura siendo el profesor el moderador del debate y el encargado de resolver cuestiones incorrectas.

\subsection{Preparación de la clase mediante GOOGLE CLASSROOM}

En primer lugar el profesor realizó una guía didáctica con los temas a desarrollar en cada una de las diferentes sesiones de la asignatura. En este caso se optó por la realización de videos explicativos de cada uno de los tema a impartir, se optó por este sistema ya que se pensó que es la forma más amena de interacción con el alumnado, además de uno de los mejores complementos para la clase invertida (Gálvez, 2015). En los videos grabados por el profesor de la asignatura se tratan aspectos fundamentales que se pondrán en práctica en las sesiones presenciales. Cada vídeo grabado tiene una duración inferior a los 12 minutos.

Una vez realizada la guía didáctica y grabados los vídeos de cada una de las sesiones programadas se optó por utilizar la plataforma GOOGLE CLASSROOM como sistema de interacción entre alumno y profesor. Para ello en primer lugar el profesor debe registrarse en la plataforma de GOOGLE CLASSROOM, figura 1 .

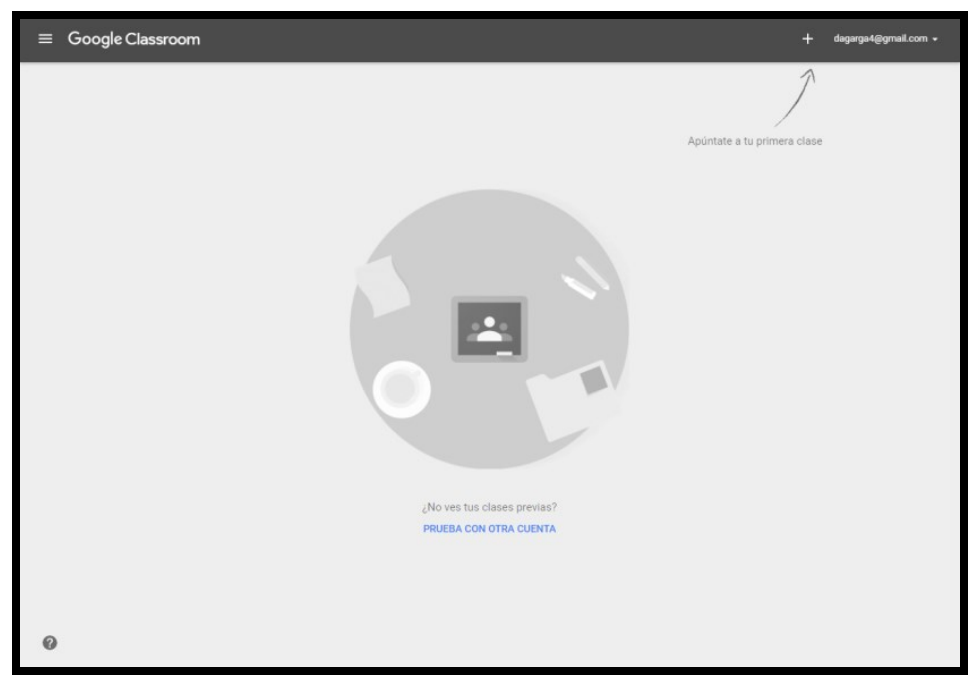

Fig. 1 Registro en la plataforma GOOGLE CLASSROOM

Una vez registrado en la plataforma se creó la clase, en este caso "Tratamiento de Residuos" una vez creada la primera clase se puede modificar el aspecto estético de la plataforma (imagen de la plataforma y colores). Una vez creada la clase, el profesor proporcionó el código de la clase (el cual es suministrado por la plataforma) a los alumnos de la asignatura. Estos desde cualquier ordenador pueden entrar a la clase creada introduciendo el código proporcionado, figura 2, permitiendo acceder a todo el material docente disponible. 


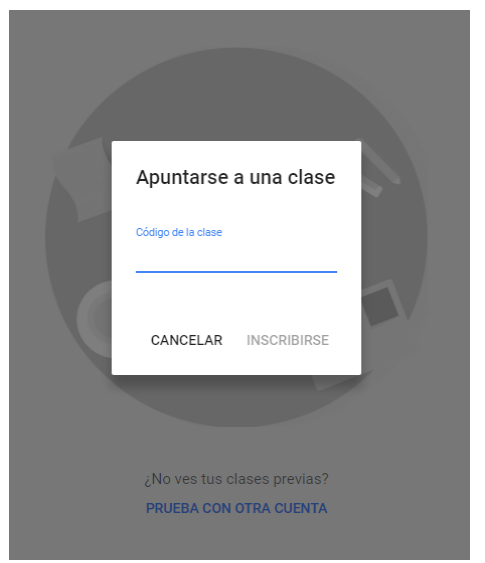

Fig. 2 Acceso a la plataforma GOOGLE CLASSROOM mediante código de acceso

Tras crear la clase el profesor subió los diferentes video de cada una de las lecciones a la plataforma, siendo notificados todos los participantes de la clase. Los videos de todos los temas fueron colgados en la plataforma una semana antes del inicio del curso académico.

Además, los videos pueden consultarse en los siguientes enlaces:

https://www.youtube.com/watch?v=MBJ7ExmVJvo\&t=84s

https://www.youtube.com/watch?v=J3qN6hRObaQ

https://www.youtube.com/watch?v=MRO9-shc3ks\&t=88s

https://www.youtube.com/watch?v=Ji9J6TQUfng\&t=350s

https://www.youtube.com/watch?v= Jxwhn6hOis

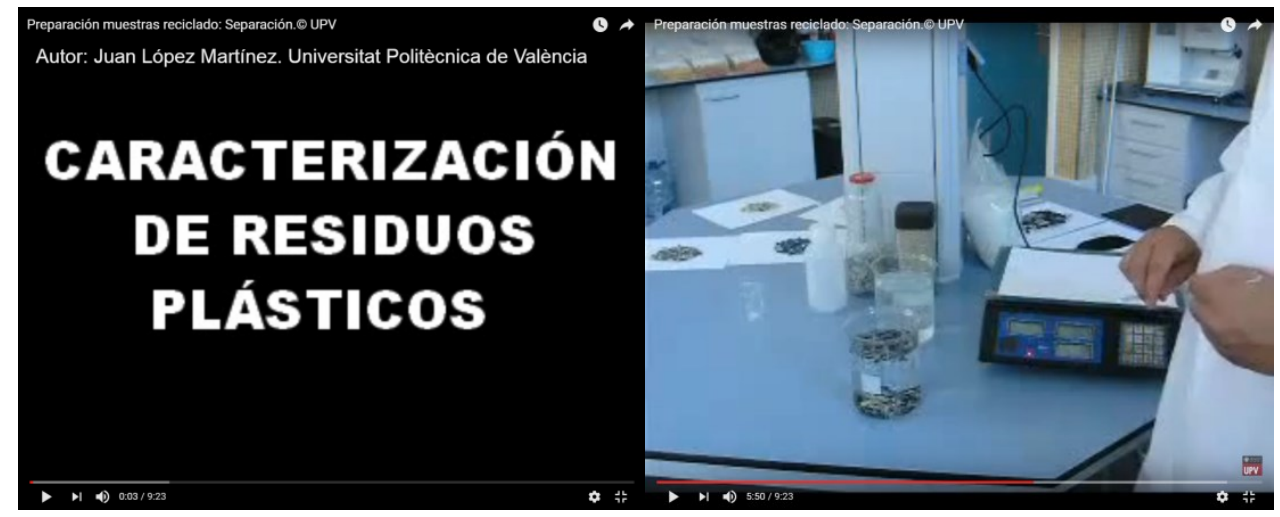

Fig. 3 Ejemplo de video realizado por el profesor y accesible desde la plataforma GOOGLE CLASSROOM

\subsection{Aplicación de la metodología de clase inversa}

La experiencia de clase inversa se realizó a lo largo del curso 2016/2017 a los 17 alumnos de la asignatura de "Tratamiento de Residuos" de cuarto curso del Grado de Ingeniería Química para el desarrollo de las prácticas de laboratorio. Inicialmente los alumnos se dividieron en grupos de 3-4 personas, en cada sesión de prácticas los grupos tenían asignados videos diferentes, un total de 5 vídeos para las 5 sesiones prácticas. Los alumnos tras la visualización de los vídeos pondrían en práctica los conocimientos adquiridos en el laboratorio que estaba organizado en tareas multipuesto. Tras la experiencia adquirida en otros años, se optó por el desarrollo de clase invertida unida a las prácticas organizadas en multipuesto para evitar que el profesor sea quien explica y realiza la práctica siendo el alumno un mero espectador que solamente anota resultados sin intervenir en el desarrollo de la práctica y manejo de los equipos. 
Para aplicar este tipo de metodología los alumnos debían visualizar el vídeo correspondiente a cada tema disponible en la plataforma GOOGLE CLASSROOM antes de asistir a las sesiones prácticas de la asignatura, cada grupo tenía asignado un vídeo diferente antes de cada sesión práctica para el correcto desarrollo de las tareas multipuesto. Además, los alumnos una vez finalizado la visualización del vídeo debían de realizar un test de autoevaluación, el cual fue preparado a través de la plataforma de GOOGLE, y que será muy similar al examen de evaluación final planteado para la asignatura. Este examen de autoevaluación no tiene valor en la nota final, únicamente sirve al alumno para saber qué aspectos necesitan reforzar antes de asistir a clase o poder plantear en clase todas las dudas relacionadas con el tema.

La primera parte de las sesiones presenciales estuvieron dedicadas a la resolución y aclaración de posibles dudas en el proceso de autoaprendizaje de los alumnos. El resto de la clase fue principalmente prácticas en laboratorio multipuesto donde se ponía en práctica todo lo aprendido con el trabajo previo en casa de forma colaborativa, convirtiéndose el profesor en un guía de prácticas sin intervenir si no es necesario, solo para dudas puntuales o por el mal uso de equipos. Las prácticas se desarrollaron en grupos de 3-4 personas que trabajaron autonomamente pasando secuencialmente por las cinco prácticas programas durante las diferentes sesiones de prácticas.

\subsection{Evaluación de la asignatura}

Una vez finalizadas todas las clases presenciales el proceso de evaluación consistió en dos partes. Una de ellas fue un test de evaluación acerca de los conceptos fundamentales visto a lo largo de la asignatura. Dicha evaluación consistió en 40 preguntas tipo test de verdadero o falso. Dicho test se realizó a través de la plataforma GOOGLE CLASSROOM y el resultado aparecía directamente una vez finalizado el test. El $60 \%$ de la nota de la asignatura se obtuvo a partir de informes en los que se le pedía al alumno que describieran las tareas realizadas en las sesiones prácticas.

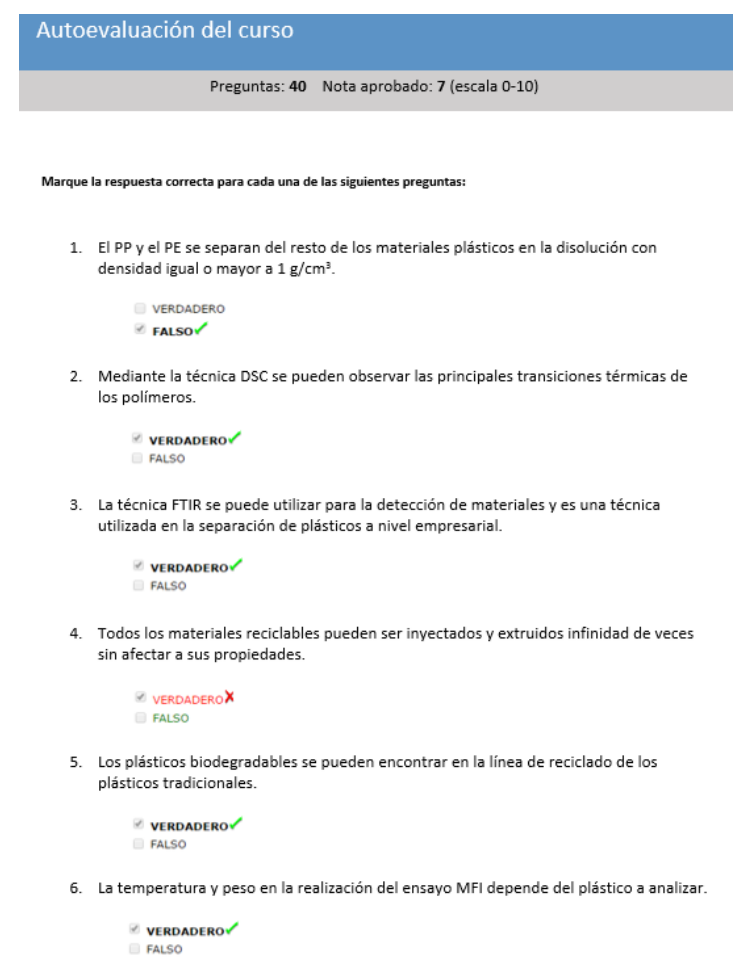

Fig. 4 Ejemplo de autoevaluación de la asignatura "Tratamiento de Residuos" 


\section{Resultados}

En la asignatura "Tratamiento de Residuos" impartida mediante la metodología de clase inversa participaron 16 de los 17 alumnos matriculados. De media, 14 alumnos habían visualizado previamente los videos sugeridos por el profesor antes de las clases prácticas, tal y como se había pedido para poder realizar correctamente la actividad, lo que supone la participación activa del 87,5\% de los alumnos. Así mismo, se planteó a los alumnos realizar de forma anónima una evaluación de la metodología empleada en la asignatura, como es la aplicación de la clase invertida apoyada con vídeos docentes a través de la plataforma GOOGLE CLASSROOM, para ello se utilizó un cuestionario realizado por el profesor y disponible en la plataforma al finalizar el test de evaluación de la asignatura.

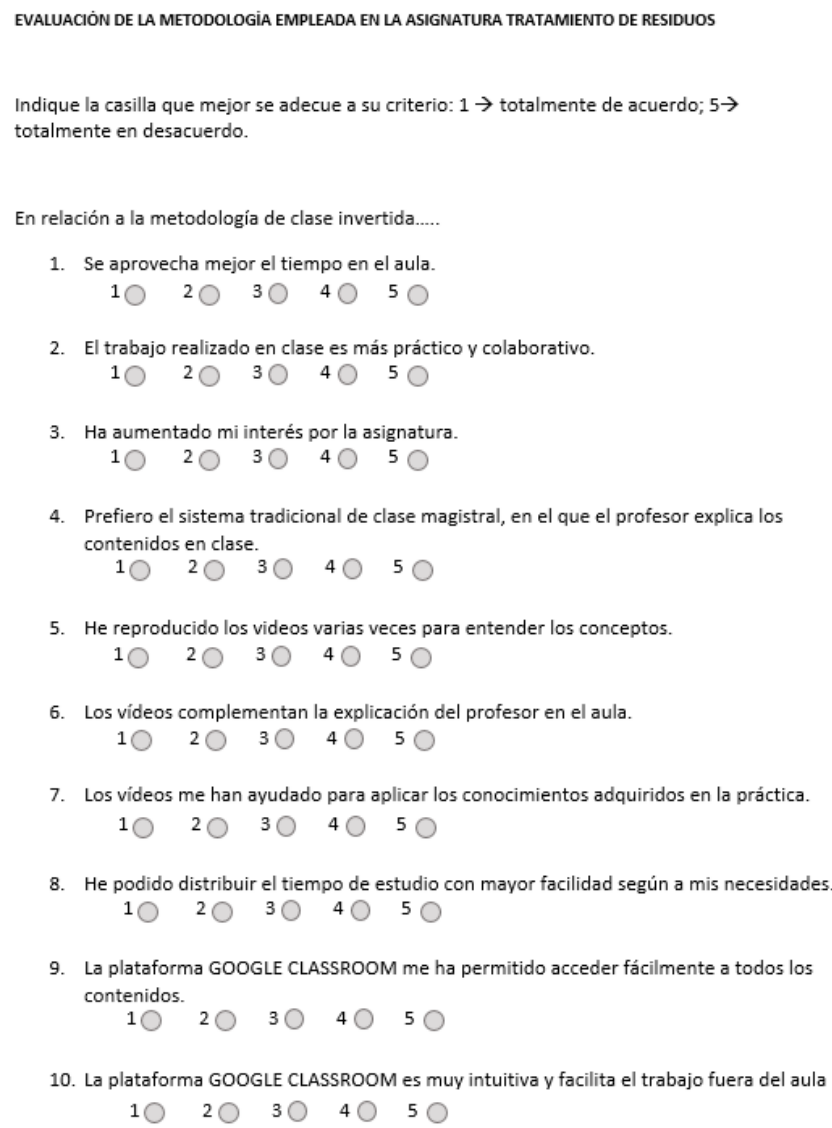

Fig. 5 Plantilla utilizada para la evaluación de la metodología clase inversa utilizando GOOGLE CLASSROOM

La calificación media obtenida por los alumnos en el cuestionario final de autoevaluación de la asignatura y que corresponde con el $40 \%$ del total de la nota de la asignatura fue de $8,4 \pm 1,6$ puntos sobre un máximo de 10 puntos. La calificación media del examen escrito tipo test de la asignatura en el curso 2015-2016

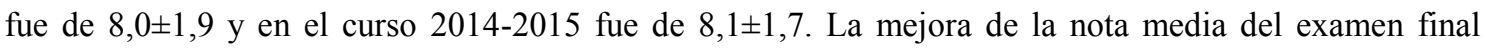
puede ser debida a que a lo largo del curso los alumnos han tenido la posibilidad de realizar diferentes test propuestos después de la visualización de los diferentes videos y plantear en clase las dudas surgidas durante el visionado del video y/o la realización del test. 


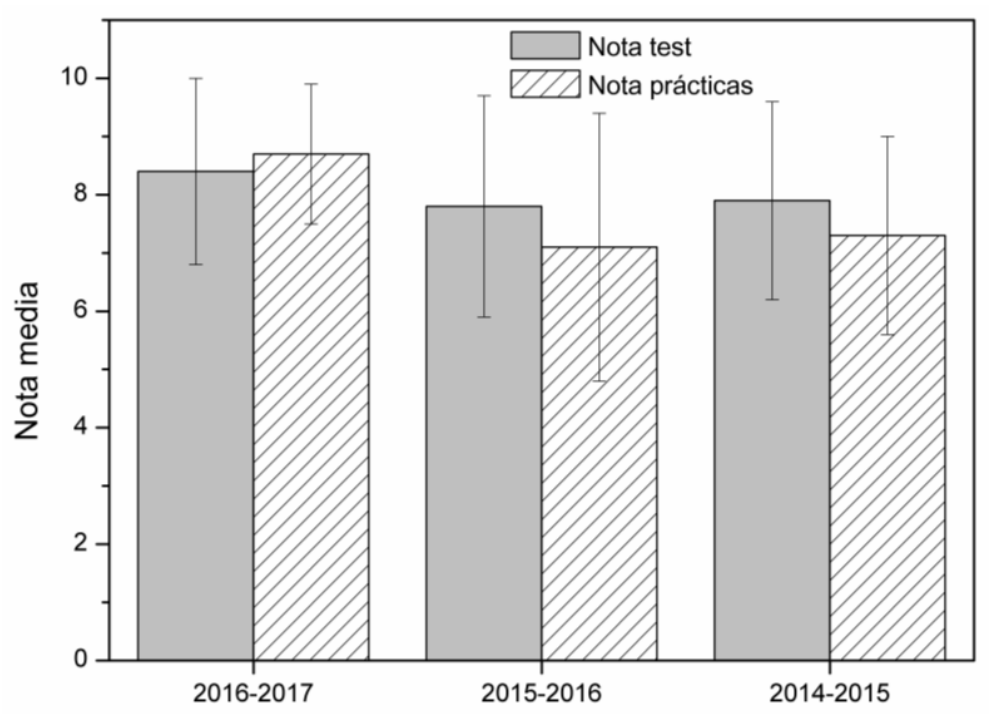

Fig. 6. Nota media de la asignatura "Tratamiento de residuos" en diferentes cursos académicos.

Por otra parte, el trabajo desarrollado durante las prácticas por los alumnos se ha visto influenciada positivamente por la metodología empleada durante la asignatura, clase inversa más tarea multipuesto, ya que los alumnos han podido trabajar en pequeños grupos de forma autónoma y manejando ellos los equipos, generando una mayor predisposición y motivación del alumnado que se ha reflejado en la mejora de los informes de prácticas y de la nota final. La nota media de prácticas obtenida en el curso 2016-2017 ha sido de $8,7 \pm 1,2$ puntos sobre 10 , que comparado con otros años supone un importante incremento, tal y como se puede observar en la figura 6, ya que en el curso 2015-2016 la nota media fue de 7,1 $\pm 2,3$ y en el curso 2014-2015 la nota media fue de 7,3 $\pm 1,7$. El incremento en la nota de prácticas puede ser debido a que los alumnos han participado activamente en el desarrollo de estas, consolidando los conceptos adquiridos en las sesiones teóricas y en los videos visualizados.

En cuanto a la evaluación por parte de los alumnos sobre la metodología seguida en el desarrollo de la asignatura, la cual se realizó mediante encuesta anónima y que realizaron 15 de los 17 alumnos matriculados, los resultados obtenidos se pueden apreciar en la tabla 1. El 73,3\% de los encuestados (11 alumnos) estaban completamente de acuerdo con la afirmación: se aprovecha mejor el tiempo en el aula. El 60\% de los alumnos ( 9 alumnos) estaban completamente de acuerdo con la afirmación: el trabajo realizado en clase es más práctico y colaborativo. El 26,7\% de los encuestados (4 alumnos) estaban completamente de acuerdo con la afirmación: ha aumentado mi interés por la asignatura; y el 40\% (6 alumnos) estaban bastante de acuerdo. El 66,7\% de los encuestados (10 alumnos) no estaban nada de acuerdo con la afirmación: prefiero el sistema tradicional de clase magistral, en el que el profesor explica los contenidos de la clase. El 66,7 \% de los encuestados (10 alumnos) están completamente de acuerdo con la afirmación: los vídeos complementan la explicación del profesor en el aula. El 60\% de los alumnos (9 alumnos) están completamente de acuerdo con la afirmación: los videos me han ayudado para aplicar los conocimientos adquiridos en la práctica. El 80\% de los alumnos (12 alumnos) están completamente de acuerdo con la afirmación: la plataforma GOOGLE CLASSROOM me ha permitido acceder fácilmente a todos los contenidos. El 73,3\% de los encuestados están de acuerdo con la afirmación: la plataforma GOOGLE CLASSROOM es muy intuitiva y facilita el trabajo fuera del aula. 
Tabla 1. Resultados de evaluación de la metodología

\begin{tabular}{|c|c|c|c|c|c|}
\hline \multirow[b]{2}{*}{ Según la afirmación: } & \multicolumn{5}{|c|}{$\mathrm{N}^{0}$ de alumnos que han contestado } \\
\hline & 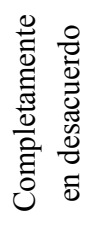 & 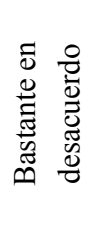 & 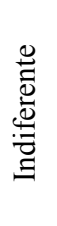 & 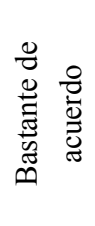 & 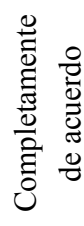 \\
\hline Se aprovecha mejor el tiempo en el aula & 0 & 0 & 1 & 3 & 11 \\
\hline El trabajo realizado en clase es más práctico y colaborativo & 0 & 0 & 2 & 4 & 9 \\
\hline Ha aumentado mi interés por la asignatura & 0 & 1 & 3 & 6 & 4 \\
\hline $\begin{array}{l}\text { Prefiero el sistema tradicional de clase magistral, en el que } \\
\text { el profesor explica los contenidos en clase }\end{array}$ & 10 & 5 & 0 & 0 & 0 \\
\hline $\begin{array}{l}\text { He reproducidos los videos varias veces para entender los } \\
\text { conceptos }\end{array}$ & 3 & 1 & 5 & 3 & 3 \\
\hline $\begin{array}{l}\text { Los vídeos complementan la explicación del profesor en el } \\
\text { aula }\end{array}$ & 0 & 0 & 1 & 4 & 10 \\
\hline $\begin{array}{l}\text { Los vídeos me han ayudado para aplicar los conocimientos } \\
\text { adquiridos en la práctica }\end{array}$ & 0 & 0 & 1 & 5 & 9 \\
\hline $\begin{array}{l}\text { He podido distrubuir el tiempo de estudio con mayor } \\
\text { facilidad según mis necesidades }\end{array}$ & 1 & 1 & 3 & 5 & 5 \\
\hline $\begin{array}{l}\text { La plataforma GOOGLE CLASSROOM me ha permitido } \\
\text { acceder fácilmente a todos los contenidos }\end{array}$ & 0 & 0 & 0 & 3 & 12 \\
\hline $\begin{array}{l}\text { La plataforma GOOGLE CLASSROOM es muy intuitiva y } \\
\text { facilita el trabajo fuera del aula }\end{array}$ & 0 & 0 & 0 & 4 & 11 \\
\hline
\end{tabular}

\section{Conclusiones}

La aplicación de la metodología de aula invertida en la asignatura "Tratamiento de Residuos" ha sido beneficiosa para los alumnos, ya que ha aumentado la nota media del test de evaluación y de los informes de prácticas de la asignatura, respecto a las notas obtenidas en otros cursos académicos. Además, hay que destacar la gran aceptación por parte de los alumnos, según la encuesta anónima realizada sobre la metodología aplicada en la asignatura, ya que destacan como puntos fuertes: el mejor aprovechamiento del tiempo en el aula, los videos complementan la explicación del profesor en el aula, la plataforma utilizada permite acceder fácilmente a todos los contenidos y la plataforma utilizada es intuitiva y facilita el trabajo fuera del aula.

Por tanto, destacamos que los videos docentes son un buen complemento para la aplicación del aula invertida, ya que las horas presenciales se aprovechan para resolver dudas y tener más tiempo para realizar trabajo práctico. Por otro lado hay que destacar el uso de la plataforma GOOGLE CLASSROOM, ya que es una herramienta práctica e intuitiva que permite el trabajo desde cualquier dispositivo.

Dada la buena experiencia obtenida con la implementación de esta metodología, no solo por la mejora en la nota obtenida por los alumnos sino también por la motivación extra que han presentado, se pretende seguir con esta metodología en años sucesivos e incluso aplicarla a otras asignaturas. 


\section{Referencias}

BARRERA, A.G. (2013). El aula inversa: cambiando la respuesta a las necesidades de los estudiantes. Avances en Supervisión Educativa.

BERGMANN, J and SAMS, A. (2012). Flip your classroom: Reach every student in every class every day. International Society for Technology in Education.

GALVEZ, A. and GARCIA A. (2015). Uso del vídeo docente para la clase invertida: evaluación, ventajas e inconvenientes. Obtido em.

JORDAN-LLUCH, C., PEÑALVER, M.J.P., and SANABRIA-CODESAL, E (2014)., Flipped Classroom: Reflexiones y opiniones de los implicados. Jornadas de Innovación Educativa y docencia en Red de la Universitat Politècnica de València.

LÓPEZ RODRÍGUEZ, D., et al. (2016). Elaboración de material para la realización de experiencias de clase inversa (flipped classroom).

TOURÓN, J., SANTIAGO, R., and DIEZ, A. (2014). The Flipped Classroom: Cómo convertir la escuela en un espacio de aprendizaje. Grupo Océano. 